\title{
Actuator Saturation Constrained Fuzzy Control for Discrete Stochastic Fuzzy Systems with Multiplicative Noises
}

\author{
Wen-Jer Chang, Cheung-Chieh Ku, and Hao-Jie Liang \\ Department of Marine Engineering, National Taiwan Ocean University, Keelung 202, Taiwan \\ Correspondence should be addressed to Wen-Jer Chang; wjchang@mail.ntou.edu.tw
}

Received 18 August 2013; Revised 3 November 2013; Accepted 3 November 2013

Academic Editor: Hui Zhang

Copyright ( 2013 Wen-Jer Chang et al. This is an open access article distributed under the Creative Commons Attribution License, which permits unrestricted use, distribution, and reproduction in any medium, provided the original work is properly cited.

\begin{abstract}
This paper deals with the fuzzy controller design problem for discrete-time Takagi-Sugeno (T-S) fuzzy systems with multiplicative noises. Using the Lyapunov stability theory and Itô formula, the sufficient conditions are derived to guarantee the stability of the closed-loop nonlinear stochastic systems subject to actuator saturation. Based on the Parallel Distributed Compensation (PDC) concept, the fuzzy controller can be obtained to stabilize the T-S fuzzy models with multiplicative noises by combining the same membership functions of plants and desired state feedback gains. In order to illustrate the availability and practicability of proposed fuzzy controller design approach, the numerical simulations for the nonlinear truck-trailer system are given to demonstrate the applications of this paper.
\end{abstract}

\section{Introduction}

In recent years, there has been an increasing interest in the fuzzy control of nonlinear systems. Some works have studied the stability and the stabilization of closed-loop fuzzy systems. Specially, these approaches are studied for the T-S fuzzy models which are described by a set of fuzzy "IF-THEN" rules with fuzzy sets in the antecedent and dynamic systems in the consequent part. The subsystems are considered as local linear models, the aggregation of which representing the nonlinear systems. Tanaka and Sugeno [1] presented sufficient conditions for the stability of T-S models. Applying the fuzzy modeling approach, many papers [2-4] have been proposed to investigate the controller design methods of nonlinear systems. According to the discrete T-S fuzzy models, the stability analysis and synthesis have been considered in [59]. Not only control schemes [5-8] but also filter design methods [9] have been proposed for nonlinear discrete-time systems via T-S fuzzy model. In general, the stochastic signals and random parameters may exist in the real systems. It is interesting to consider the stochastic behaviors for analyzing the stability of nonlinear stochastic systems. Recently, the T-S fuzzy system with multiplicative noise term [10-16] is structured for representing the nonlinear stochastic system. In $[10,11,13,15]$, the time delay phenomenon is considered in the control problem. Besides, the fuzzy filter design problem for nonlinear stochastic systems is studied in [12]. Different from the traditional additive noise, multiplicative noise is more practical since it allows the statistical description of the noise to be unknown a prior but depends on the control and state solution. Therefore, the T-S fuzzy model with multiplicative noises is considered in this paper to represent the nonlinear stochastic systems.

The presence of actuator saturation imposes additional constraints on the analysis and synthesis of control systems. Addressing actuator saturation has been well recognized to be practically imperative, yet it brings theoretically challenging. Physical capacity of the actuator is limited and the actuator saturation may severely degrade the performance of the closed-loop systems. The actuator saturation usually leads to a large overshoot, induces a limit cycle, and even makes the otherwise stable closed-loop system unstable. This is reflected in the large body of literature on linear and nonlinear systems in the presence of actuator saturation (see, e.g. [17-24]). Recently, some fuzzy control approaches for nonlinear systems subject to actuator saturation were investigated in [1924]. Most of recent papers studied the fuzzy controller design problem of continuous-time nonlinear systems subject to actuator saturation. However, few papers [21] have been 
proposed to investigate the similar problems for discrete nonlinear systems, especially for discrete nonlinear stochastic systems. In general, the saturation function is characterized in terms of convex hull of some linear combinations of linear function and saturation function [19]. However, the number of stability conditions increases dramatically with the number of control inputs. In [22], the saturation function is formulated inside a specific nonlinear saturation sector that provides less stability condition numbers. The saturation function formulated in [22] has not been employed to deal with the actuator saturation constrained control problem for the discrete-time T-S fuzzy model with multiplicative noises. Thus, the motivation of this paper is to discuss fuzzy control problem of discrete-time nonlinear stochastic system with multiplicative noises and actuator saturation. The actuator saturation function formulated in [22] is employed in this paper to design a stable fuzzy controller.

To deal with a performance constrained control problem for the nonlinear T-S fuzzy stochastic systems, a fuzzy controller design subject to actuator saturation is investigated in this paper. Based on the PDC concept [25], the fuzzy controller can be obtained by combining the same membership functions of plant and linear feedback gains of subsystems. Thus, the overall system input can be blended by these linear feedback gains. Usually, the sufficient conditions are derived into Linear Matrix Inequality (LMI) forms [26] which can be calculated by optimal convex algorithm [27] for finding a common definite matrix and feedback gains. According to the actuator saturation function formulated in [22], the contribution of this paper is to develop a PDCbased fuzzy controller design methodology for guaranteeing the stability of discrete-time T-S fuzzy models with multiplicative noises. In order to demonstrate the applicability and effectiveness of the proposed design approach, the fuzzy controller design problem of a truck-trailer system [28] subject to actuator saturation is discussed in this paper.

The organization of this paper is structured as follows. The structure of T-S fuzzy model with multiplicative noises is introduced in Section 2. In Section 3, a fuzzy controller design methodology is developed by using the concept of PDC and the Lyapunov stability criterion. Through applying the proposed fuzzy controller design approach, simulation results for the nonlinear stochastic truck-trailer systems are demonstrated in Section 4. Finally, some conclusions are proposed in Section 5.

\section{System Descriptions and Problem Statements}

The T-S fuzzy model is described by fuzzy IF-THEN rules, which represent local linear input-output relations of the nonlinear systems. This section outlines the mathematical model of the T-S fuzzy model with multiplicative noise for the discrete-time nonlinear stochastic systems. The $i$-th rule of T-S fuzzy model is introduced in the following form.

\subsection{Plant Part}

Rule $i$

$$
\begin{gathered}
\text { IF } \quad z_{1}(k) \text { is } M_{i 1} \cdots \text { and } z_{p}(k) \text { is } M_{i p} \\
\text { THEN } \quad x(k+1)=\mathbf{A}_{i} x(k)+\mathbf{B}_{i} \bar{u}(k) \\
+\left(\overline{\mathbf{A}}_{i} x(k)+\overline{\mathbf{B}}_{i} \bar{u}(k)\right) w(k),
\end{gathered}
$$

where $\mathbf{A}_{i} \in \mathbb{R}^{n \times n}, \mathbf{B}_{i} \in \mathbb{R}^{n \times m}, \overline{\mathbf{A}}_{i} \in \mathbb{R}^{n \times n}$, and $\overline{\mathbf{B}}_{i} \in$ $\mathbb{R}^{n \times m}$ are constant matrices, $i=1,2, \ldots, r$ and $r$ is the number of fuzzy rules, and $z_{1}(k), z_{2}(k), \ldots, z_{p}(k)$ are premise variables. Besides, $x(k) \in \mathbb{R}^{n}$ denotes the state vector, $\bar{u}(k)=$ $\operatorname{sat}(u(k)) \in \mathbb{R}^{m}$ denotes the saturating control input, and $w(k)$ is a scalar zero mean white noise with variance one.

In this paper, the saturating actuator is defined as follows:

$$
\bar{u}_{k}(k)=\operatorname{sat}\left(u_{k}\right)= \begin{cases}u_{k L} & \text { if } u_{k}<u_{k L} \\ u_{k} & \text { if } u_{k L} \leq u_{k} \leq u_{k H} \\ u_{k H} & \text { if } u_{k H}<u_{k},\end{cases}
$$

where $u_{k L}<0<u_{k H}$ and $k=1,2, \ldots, m$.

Given a pair of $(x(k), \bar{u}(k))$, the final output of the fuzzy system is inferred as follows:

$$
\begin{aligned}
x(k+1) & \\
= & \left(\sum_{i=1}^{r} \omega_{i}(z(k))\right. \\
& \left.\times\left\{\mathbf{A}_{i} x(k)+\mathbf{B}_{i} \bar{u}(k)+\left(\overline{\mathbf{A}}_{i} x(k)+\overline{\mathbf{B}}_{i} \bar{u}(k)\right) w(k)\right\}\right) \\
& \times\left(\sum_{i=1}^{r} \omega_{i}(z(k))\right)^{-1} \\
= & \sum_{i=1}^{r} h_{i}(z(k))\left\{\mathbf{A}_{i} x(k)+\mathbf{B}_{i} \bar{u}(k)\right. \\
& \left.+\left(\overline{\mathbf{A}}_{i} x(k)+\overline{\mathbf{B}}_{i} \bar{u}(k)\right) w(k)\right\},
\end{aligned}
$$

where $z(k)=\left[z_{1}(k) z_{2}(k) \cdots z_{p}(k)\right]^{T}, h_{i}(z(k))=\omega_{i}(z(k)) /$ $\sum_{i=1}^{r} \omega_{i}(z(k))$ and $\omega_{i}(z(k))=\prod_{j=1}^{p} M_{i j}\left(z_{j}(k)\right)$. Note that $h_{i}(z(k)) \geq 0$ and $\sum_{i=1}^{r} h_{i}(z(k))=1$.

Considering the saturating actuator, one can formulate the following inequality from relations of $\bar{u}_{k}(k)$ defined in (2):

$$
\|u(k)\| \geq\|\bar{u}(k)\| .
$$

The following inequality can be derived from the inequality (4) and Remark 1 of [22]:

$$
\frac{1-\varepsilon}{2}\|u(k)\| \geq\left\|\bar{u}(k)-\frac{1+\varepsilon}{2} u(k)\right\|,
$$

where $0<\varepsilon<1$. The sector parameter $\varepsilon$ can be used to guarantee that the saturation map sat is inside the sector 
$(\varepsilon, 1)$. The inequality (5) can be arranged by $u_{k H} \geq \varepsilon u_{k}$ and $u_{k L} \leq \varepsilon u_{k}$ as follows:

$$
\frac{u_{k L}}{\varepsilon} \leq u_{k} \leq \frac{u_{k H}}{\varepsilon}, \quad k=1,2, \ldots, m .
$$

In this paper, one can find that if $u_{k H}=-u_{k L}$ is set, then one has

$$
\left|u_{k}\right| \leq \frac{u_{k H}}{\varepsilon}
$$

Expanding the inequality (5), one can obtain the following inequality:

$$
\begin{aligned}
& \left(\bar{u}(k)-\frac{1+\varepsilon}{2} u(k)\right)^{T}\left(\bar{u}(k)-\frac{1+\varepsilon}{2} u(k)\right) \\
& \leq\left(\frac{1-\varepsilon}{2}\right)^{2} u^{T}(k) u(k) .
\end{aligned}
$$

The inequality (8) is an important basis in the following derivations of this paper. According to the sector parameter $\varepsilon$, the state equation (3) can be rewritten as

$$
\begin{aligned}
x(k+1) & \\
=\sum_{i=1}^{r} h_{i}(z(k)) & \\
& \times\left\{\mathbf{A}_{i} x(k)+\mathbf{B}_{i} \bar{u}(k)+\overline{\mathbf{A}}_{i} w(k) x(k)+\overline{\mathbf{B}}_{i} w(k) \bar{u}(k)\right\} \\
=\sum_{i=1}^{r} h_{i}(z(k)) & \left\{\mathbf{A}_{i} x(k)+\mathbf{B}_{i}\left(\frac{1+\varepsilon}{2}\right) u(k)\right. \\
& +\mathbf{B}_{i}\left(\bar{u}(k)-\frac{1+\varepsilon}{2} u(k)\right) \\
& +\overline{\mathbf{A}}_{i} w(k) x(k)+\overline{\mathbf{B}}_{i} w(k)\left(\frac{1+\varepsilon}{2}\right) u(k) \\
& \left.+\overline{\mathbf{B}}_{i} w(k)\left(\bar{u}(k)-\frac{1+\varepsilon}{2} u(k)\right)\right\} .
\end{aligned}
$$

Based on the PDC concept [25], the fuzzy controller can be proposed as follows.

\subsection{Controller Part}

Rule $i$

$$
\begin{gathered}
\text { IF } \quad z_{1}(k) \text { is } M_{i 1} \cdots \text { and } z_{p}(k) \text { is } M_{i p} \\
\text { THEN } u(k)=\mathbf{K}_{i} x(k) \quad \text { for } i=1,2, \ldots, r
\end{gathered}
$$

where $\mathbf{K}_{i} \in \mathbb{R}^{m \times n}$ are constant feedback matrices. The output of PDC-based fuzzy controller (10) is determined by the summation

$$
u(k)=\frac{\sum_{i=1}^{r} \omega_{i}(z(k))\left(\mathbf{K}_{i} x(k)\right)}{\sum_{i=1}^{r} \omega_{i}(z(k))}=\sum_{i=1}^{r} h_{i}(z(k))\left(\mathbf{K}_{i} x(k)\right) .
$$

By substituting (11) into (9), one can obtain the corresponding closed-loop system as follows:

$$
\begin{aligned}
& x(k+1) \\
& =\sum_{i=1}^{r} h_{i}(z(k)) \\
& \quad \times\left\{\mathbf{A}_{i} x(k)+\mathbf{B}_{i} \bar{u}(k)+\left(\overline{\mathbf{A}}_{i} x(k)+\overline{\mathbf{B}}_{i} \bar{u}(k)\right) w(k)\right\} \\
& =\sum_{i=1}^{r} \sum_{j=1}^{r} h_{i}(z(k)) h_{j}(z(k))\left\{\mathbf{A}_{i j} x(k)+\overline{\mathbf{A}}_{i j} w(k) x(k)\right. \\
& +\mathbf{B}_{i}\left(\bar{u}(k)-\frac{1+\varepsilon}{2} \mathbf{K}_{j} x(k)\right) \\
& +\overline{\mathbf{B}}_{i} w(k) \\
& \left.\times\left(\bar{u}(k)-\frac{1+\varepsilon}{2} \mathbf{K}_{j} x(k)\right)\right\},
\end{aligned}
$$

where $\mathbf{A}_{i j}=\mathbf{A}_{i}+((1+\varepsilon) / 2) \mathbf{B}_{i} \mathbf{K}_{j}$ and $\overline{\mathbf{A}}_{i j}=\overline{\mathbf{A}}_{i}+((1+\varepsilon) / 2) \overline{\mathbf{B}}_{i} \mathbf{K}_{j}$. After arranging (12), the augmented system can be derived in the following form:

$$
\begin{aligned}
& x(k+1) \\
& =\sum_{i=1}^{r} h_{i}^{2}(z(k)) \\
& +\left\{\mathbf{A}_{i i} x(k)+\overline{\mathbf{A}}_{i i} w(k) x(k)\right. \\
& +\mathbf{B}_{i}\left(\bar{u}(k)-\frac{1+\varepsilon}{2} \mathbf{K}_{i} x(k)\right) \\
& \left.+\overline{\mathbf{B}}_{i} w(k)\left(\bar{u}(k)-\frac{1+\varepsilon}{2} \mathbf{K}_{i} x(k)\right)\right\} \\
& +2 \sum_{i=1}^{r} \sum_{i<j}^{r} h_{i}(z(k)) h_{j}(z(k)) \\
& \times\left\{\frac { 1 } { 2 } \left(\left(\mathbf{A}_{i j}+\mathbf{A}_{j i}\right) x(k)+\left(\overline{\mathbf{A}}_{i j}+\overline{\mathbf{A}}_{j i}\right) w(k) x(k)\right.\right. \\
& +\left(\mathbf{B}_{i}\left(\bar{u}(k)-\frac{1+\varepsilon}{2} \mathbf{K}_{j} x(k)\right)\right. \\
& \left.+\mathbf{B}_{j}\left(\bar{u}(k)-\frac{1+\varepsilon}{2} \mathbf{K}_{i} x(k)\right)\right) \\
& +\left(\overline{\mathbf{B}}_{i}\left(\bar{u}(k)-\frac{1+\varepsilon}{2} \mathbf{K}_{j} x(k)\right)\right. \\
& \left.\left.\left.+\overline{\mathbf{B}}_{j} w(k)\left(\bar{u}(k)-\frac{1+\varepsilon}{2} \mathbf{K}_{i} x(k)\right)\right)\right)\right\}
\end{aligned}
$$


Let an ellipsoid $\Omega_{1}$ and a positive scalar function $V(x(k))$ be defined as follows, respectively:

$$
\begin{gathered}
\Omega_{1}=\left\{x(k) \mid x^{T}(k) \mathbf{P} x(k) \leq 1\right\}, \\
V(x(k))=x^{T}(k) \mathbf{P} x(k),
\end{gathered}
$$

where $\mathbf{P} \in \mathbb{R}^{n \times n}$ denotes a positive definite matrix. The ellipsoid $\Omega_{1}$, which is inside the domain of attraction, is said to be contractively invariant [19] if the following condition can be satisfied:

$$
\Delta V(x(k))<0, \quad \forall x(k) \in \Omega_{1} \backslash\{0\} .
$$

From (7) and (11), the constraint $\left|u_{k}\right| \leq u_{k H} / \varepsilon$ can be inferred as follows:

$$
\left|\sum_{i=1}^{r} h_{i}(z(k))\left(\mathbf{K}_{i}^{(k)} x(k)\right)\right| \leq \frac{u_{k H}}{\varepsilon},
$$

where $\mathbf{K}_{i}^{(k)}$ denotes the $k$-th row of $\mathbf{K}_{i}$. It is obvious that if (16) holds with $h_{i}(z(k))=1$, then one can define the following equation:

$$
\Omega_{2}=\left\{x(k) \mid x^{T}(k)\left(\mathbf{K}_{i}^{(k)}\right)^{T}\left(\mathbf{K}_{i}^{(k)}\right) x(k) \leq\left(\frac{u_{k H}}{\varepsilon}\right)^{2}\right\} .
$$

In this paper, it is required that $x(k) \in \Omega_{1} \subset \Omega_{2}$; that is, $\Omega_{1}$ is a subset of $\Omega_{2}$. The equivalent condition for $x(k) \in \Omega_{1} \subset \Omega_{2}$ can be represented as follows:

$$
\left(\mathbf{K}_{i}^{(k)}\right) \mathbf{P}^{-1}\left(\mathbf{K}_{i}^{(k)}\right)^{T} \leq\left(\frac{u_{k H}}{\varepsilon}\right)^{2}
$$

Considering the discrete-time T-S fuzzy model with multiplicative noises (1), the purpose of this paper is to find the solutions of PDC-based fuzzy controller (11) subject to actuator saturation defined in (2). Employing the Lyapunov stability criterion and Itô formula, the stability conditions are derived in the next section. Solving these stability conditions, the feedback gains $\mathbf{K}_{i}$ of PDC-based fuzzy controller (11) can be used to stabilize the discrete-time T-S fuzzy model with multiplicative noises (1) subject to the constraint of actuator saturation (2).

\section{Stability Conditions Derivations and Fuzzy Controller Design}

In this section, the stability conditions are derived for the closed-loop system (12) subject to actuator saturation (2). Before describing the stability conditions, the Lyapunov function is defined in the following equation which satisfies $x(0) \in \Omega_{1} \subset \Omega_{2}$ and $x(k) \in \Omega_{1} \subset \Omega_{2}$, for all $k \geq 0$ :

$$
V(x(k))=x^{T}(k) \mathbf{P} x(k) .
$$

Based on the Lyapunov function (19), one can obtain the following theorem for analyzing the stability of augmented system (12).
Theorem 1. Considering the actuator saturation (2), the closed-loop system (12) is asymptotically stable if there exist matrix $\mathbf{Q}=\mathbf{Q}^{T}>0$ and feedback gains $\mathbf{K}_{i}$ such that

$$
\begin{gathered}
{\left[\begin{array}{ccc}
-\mathbf{Q} & \mathbf{Q} \mathbf{A}_{i}^{T}+\mathbf{Y}_{j}^{T} \mathbf{B}_{i}^{T} & \mathbf{Q} \overline{\mathbf{A}}_{i}^{T}+\mathbf{Y}_{j}^{T} \overline{\mathbf{B}}_{i}^{T} \\
* & -\mathbf{Q} & 0 \\
* & * & -\mathbf{Q}
\end{array}\right]<0} \\
{\left[\begin{array}{cc}
\ell & \frac{1+\varepsilon}{2} \mathbf{Y}_{i}^{(k)} \\
* & \mathbf{Q}
\end{array}\right] \geq 0}
\end{gathered}
$$

where $\mathbf{Q}=\mathbf{P}^{-1}, \mathbf{Y}_{i}=\mathbf{K}_{i} \mathbf{Q}, \mathbf{Y}_{i}^{(k)}=\mathbf{K}_{i}^{(k)} \mathbf{Q}$, and $\ell=$ $((1+\varepsilon) / 2)^{2}\left(u_{k H} / \varepsilon\right)^{2}$ and $*$ denotes the transposed element in the symmetric position.

Proof. By choosing the Lyapunov function defined in (19), one can obtain

$$
\Delta V(x(k))=V(x(k+1))-V(x(k)) .
$$

According to the Lyapunov function (19) and the closedloop system (12), one can get

$$
\begin{aligned}
& V(x(k+1)) \\
& =x^{T}(k+1) \mathbf{P} x(k+1) \\
& =\sum_{i=1}^{r} \sum_{j=1}^{r} h_{i}(z(k)) h_{j}(z(k)) \\
& \times\left\{\left(\mathbf{A}_{i j} x(k)+\mathbf{B}_{i}\left(\bar{u}(k)-\frac{1+\varepsilon}{2} \mathbf{K}_{j} x(k)\right)\right.\right. \\
& +\overline{\mathbf{A}}_{i j} w(k) x(k) \\
& \left.+\overline{\mathbf{B}}_{i} w(k)\left(\bar{u}(k)-\frac{1+\varepsilon}{2} \mathbf{K}_{j} x(k)\right)\right)^{T} \\
& \quad \times \mathbf{P}\left(\mathbf{A}_{i j} x(k)+\mathbf{B}_{i}\left(\bar{u}(k)-\frac{1+\varepsilon}{2} \mathbf{K}_{j} x(k)\right)\right. \\
& +\overline{\mathbf{A}}_{i j} w(k) x(k)+\overline{\mathbf{B}}_{i} w(k) \\
& \left.\left.\times\left(\bar{u}(k)-\frac{1+\varepsilon}{2} \mathbf{K}_{j} x(k)\right)\right)\right\} \\
& \times\left\{\left(\mathbf{A}_{i i} x(k)+\mathbf{B}_{i}\left(\bar{u}(k)-\frac{1+\varepsilon}{2} \mathbf{K}_{i} x(k)\right)\right.\right. \\
& =\sum_{i=1}^{r} h_{i}^{2}(z(k))
\end{aligned}
$$




$$
\begin{aligned}
& \left.+\overline{\mathbf{A}}_{i i} x(k)+\overline{\mathbf{B}}_{i}\left(\bar{u}(k)-\frac{1+\varepsilon}{2} \mathbf{K}_{i} x(k)\right)\right)^{T} \\
& \times \mathbf{P}\left(\mathbf{A}_{i i} x(k)+\mathbf{B}_{i}\left(\bar{u}(k)-\frac{1+\varepsilon}{2} \mathbf{K}_{i} x(k)\right)\right. \\
& \left.\left.+\overline{\mathbf{A}}_{i i} x(k)+\overline{\mathbf{B}}_{i}\left(\bar{u}(k)-\frac{1+\varepsilon}{2} \mathbf{K}_{i} x(k)\right)\right)\right\} \\
& +2 \sum_{i=1}^{r} \sum_{i<j}^{r} h_{i}(z(k)) h_{j}(z(k)) \\
& \times\left\{\frac { 1 } { 2 } \left(\mathbf{A}_{i j} x(k)+\mathbf{B}_{i}\left(\bar{u}(k)-\frac{1+\varepsilon}{2} \mathbf{K}_{j} x(k)\right)\right.\right. \\
& +\mathbf{A}_{j i} x(k)+\mathbf{B}_{j}\left(\bar{u}(k)-\frac{1+\varepsilon}{2} \mathbf{K}_{i} x(k)\right) \\
& +\overline{\mathbf{A}}_{i j} x(k)+\overline{\mathbf{B}}_{i}\left(\bar{u}(k)-\frac{1+\varepsilon}{2} \mathbf{K}_{j} x(k)\right) \\
& \left.+\overline{\mathbf{A}}_{j i} x(k)+\overline{\mathbf{B}}_{j}\left(\bar{u}(k)-\frac{1+\varepsilon}{2} \mathbf{K}_{i} x(k)\right)\right)^{T} \\
& \times \mathbf{P}\left(\frac { 1 } { 2 } \left(\mathbf{A}_{i j} x(k)+\mathbf{B}_{i}\left(\bar{u}(k)-\frac{1+\varepsilon}{2} \mathbf{K}_{j} x(k)\right)\right.\right. \\
& +\mathbf{A}_{j i} x(k)+\mathbf{B}_{j}\left(\bar{u}(k)-\frac{1+\varepsilon}{2} \mathbf{K}_{i} x(k)\right) \\
& +\overline{\mathbf{A}}_{i j} x(k)+\overline{\mathbf{B}}_{i}\left(\bar{u}(k)-\frac{1+\varepsilon}{2} \mathbf{K}_{j} x(k)\right) \\
& \left.\left.\left.+\overline{\mathbf{A}}_{j i} x(k)+\overline{\mathbf{B}}_{j}\left(\bar{u}(k)-\frac{1+\varepsilon}{2} \mathbf{K}_{i} x(k)\right)\right)\right)\right\} \text {. }
\end{aligned}
$$

According to (8), (23), and the fact that $\mathbf{X}^{T} \mathbf{Y}+\mathbf{Y}^{T} \mathbf{X} \leq$ $\mathbf{X}^{T} \mathbf{X}+\mathbf{Y}^{T} \mathbf{Y}$, the inequality (22) can be arranged as follows:

$$
\begin{aligned}
& \Delta V(x(k)) \\
& \leq \sum_{i=1}^{r} \sum_{i<j}^{r} h_{i}(z(k)) h_{j}(z(k)) x^{T}(k) \\
& \times\left\{\frac { 1 } { 4 } \left(\mathbf{A}_{i j}^{T} \mathbf{P} \mathbf{A}_{i j}+\mathbf{A}_{i j}^{T} \mathbf{P} \mathbf{A}_{j i}+\mathbf{A}_{j i}^{T} \mathbf{P} \mathbf{A}_{i j}+\mathbf{A}_{j i}^{T} \mathbf{P} \mathbf{A}_{j i}\right.\right. \\
&+\mathbf{A}_{i j}^{T} \mathbf{P B}_{i}\left(\frac{1-\varepsilon}{2} \mathbf{K}_{j}\right)+\mathbf{A}_{i j}^{T} \mathbf{P} \mathbf{B}_{j}\left(\frac{1-\varepsilon}{2} \mathbf{K}_{i}\right) \\
&+\mathbf{A}_{j i}^{T} \mathbf{P} \mathbf{B}_{i}\left(\frac{1-\varepsilon}{2} \mathbf{K}_{j}\right)+\mathbf{A}_{j i}^{T} \mathbf{P} \mathbf{B}_{j}\left(\frac{1-\varepsilon}{2} \mathbf{K}_{i}\right) \\
&+\left(\frac{1-\varepsilon}{2} \mathbf{K}_{j}\right)^{T} \mathbf{B}_{i}^{T} \mathbf{P A}_{i j}+\left(\frac{1-\varepsilon}{2} \mathbf{K}_{j}\right)^{T} \mathbf{B}_{i}^{T} \mathbf{P} \mathbf{A}_{j i} \\
&+\left(\frac{1-\varepsilon}{2} \mathbf{K}_{i}\right)^{T} \mathbf{B}_{j}^{T} \mathbf{P A}_{i j}+\left(\frac{1-\varepsilon}{2} \mathbf{K}_{i}\right)^{T} \mathbf{B}_{j}^{T} \mathbf{P} \mathbf{A}_{j i} \\
&+\left(\frac{1-\varepsilon}{2} \mathbf{K}_{j}\right)^{T} \mathbf{B}_{i}^{T} \mathbf{P} \mathbf{B}_{i}\left(\frac{1-\varepsilon}{2} \mathbf{K}_{j}\right) \\
&+\left(\frac{1-\varepsilon}{2} \mathbf{K}_{j}\right)^{T} \mathbf{B}_{i}^{T} \mathbf{P B}_{j}\left(\frac{1-\varepsilon}{2} \mathbf{K}_{i}\right)
\end{aligned}
$$

$$
\begin{aligned}
& +\left(\frac{1-\varepsilon}{2} \mathbf{K}_{i}\right)^{T} \mathbf{B}_{j}^{T} \mathbf{P B}_{i}\left(\frac{1-\varepsilon}{2} \mathbf{K}_{j}\right) \\
& +\left(\frac{1-\varepsilon}{2} \mathbf{K}_{i}\right)^{T} \mathbf{B}_{j}^{T} \mathbf{P B}_{j}\left(\frac{1-\varepsilon}{2} \mathbf{K}_{i}\right) \\
& +\overline{\mathbf{A}}_{i j}^{T} \mathbf{P} \overline{\mathbf{A}}_{i j}+\overline{\mathbf{A}}_{i j}^{T} \mathbf{P} \overline{\mathbf{A}}_{j i}+\overline{\mathbf{A}}_{j i}^{T} \mathbf{P} \overline{\mathbf{A}}_{i j}+\overline{\mathbf{A}}_{j i}^{T} \mathbf{P} \overline{\mathbf{A}}_{j i} \\
& +\overline{\mathbf{A}}_{i j}^{T} \mathbf{P} \overline{\mathbf{B}}_{i}\left(\frac{1-\varepsilon}{2} \mathbf{K}_{j}\right)+\overline{\mathbf{A}}_{i j}^{T} \mathbf{P} \overline{\mathbf{B}}_{j}\left(\frac{1-\varepsilon}{2} \mathbf{K}_{i}\right) \\
& +\overline{\mathbf{A}}_{j i}^{T} \mathbf{P} \overline{\mathbf{B}}_{i}\left(\frac{1-\varepsilon}{2} \mathbf{K}_{j}\right)+\overline{\mathbf{A}}_{j i}^{T} \mathbf{P} \overline{\mathbf{B}}_{j}\left(\frac{1-\varepsilon}{2} \mathbf{K}_{i}\right) \\
& +\left(\frac{1-\varepsilon}{2} \mathbf{K}_{j}\right)^{T} \overline{\mathbf{B}}_{i}^{T} \mathbf{P} \overline{\mathbf{A}}_{i j}+\left(\frac{1-\varepsilon}{2} \mathbf{K}_{j}\right)^{T} \overline{\mathbf{B}}_{i}^{T} \mathbf{P} \overline{\mathbf{A}}_{j i} \\
& +\left(\frac{1-\varepsilon}{2} \mathbf{K}_{i}\right)^{T} \overline{\mathbf{B}}_{j}^{T} \mathbf{P} \overline{\mathbf{A}}_{i j}+\left(\frac{1-\varepsilon}{2} \mathbf{K}_{i}\right)^{T} \overline{\mathbf{B}}_{j}^{T} \mathbf{P} \overline{\mathbf{A}}_{j i} \\
& +\left(\frac{1-\varepsilon}{2} \mathbf{K}_{j}\right)^{T} \overline{\mathbf{B}}_{i}^{T} \mathbf{P} \overline{\mathbf{B}}_{i}\left(\frac{1-\varepsilon}{2} \mathbf{K}_{j}\right) \\
& +\left(\frac{1-\varepsilon}{2} \mathbf{K}_{j}\right)^{T} \overline{\mathbf{B}}_{i}^{T} \mathbf{P} \overline{\mathbf{B}}_{j}\left(\frac{1-\varepsilon}{2} \mathbf{K}_{i}\right) \\
& +\left(\frac{1-\varepsilon}{2} \mathbf{K}_{i}\right)^{T} \overline{\mathbf{B}}_{j}^{T} \mathbf{P} \overline{\mathbf{B}}_{i}\left(\frac{1-\varepsilon}{2} \mathbf{K}_{j}\right)+\left(\frac{1-\varepsilon}{2} \mathbf{K}_{i}\right)^{T} \\
& \left.\left.\times \overline{\mathbf{B}}_{j}^{T} \mathbf{P} \overline{\mathbf{B}}_{j}\left(\frac{1-\varepsilon}{2} \mathbf{K}_{i}\right)\right)\right\} x(k)-x^{T}(k) \mathbf{P} x(k) \\
& =\sum_{i=1}^{r} \sum_{i<j}^{r} h_{i}(z(k)) h_{j}(z(k)) x^{T}(k) \mathbf{P} \Theta_{i j} \mathbf{P} x(k),
\end{aligned}
$$

where

$$
\begin{aligned}
\Theta_{i j}=\mathbf{P}^{-1}( & \left(\mathbf{A}_{i}+\mathbf{B}_{i} \mathbf{K}_{j}\right)^{T} \mathbf{P}\left(\mathbf{A}_{i}+\mathbf{B}_{i} \mathbf{K}_{j}\right) \\
& \left.+\left(\overline{\mathbf{A}}_{i}+\overline{\mathbf{B}}_{i} \mathbf{K}_{j}\right)^{T} \mathbf{P}\left(\overline{\mathbf{A}}_{i}+\overline{\mathbf{B}}_{i} \mathbf{K}_{j}\right)-\mathbf{P}\right) \mathbf{P}^{-1} .
\end{aligned}
$$

Using the Schur complement [26], the condition (20) is equivalent to $\Theta_{i j}<0$. Obviously, if the condition (20) of Theorem 1 is satisfied, then $\Delta V(x(k))<0$ can be obtained from (25). Therefore, the condition (20) is provided for guaranteeing the asymptotical stability of the closed-loop system (12). Additionally, using the Schur complement [26], the following relation can be obtained from (18):

$$
\left[\begin{array}{cc}
\ell_{2} & \frac{1+\varepsilon}{2} \mathbf{Y}_{i}^{(k)} \\
* & \mathbf{Q}
\end{array}\right] \geq 0
$$

where $\mathbf{Y}_{i}^{(k)}=\mathbf{K}_{i}^{(k)} \mathbf{Q}$ and $\ell_{2}=((1+\varepsilon) / 2)^{2}\left(u_{k H} / \varepsilon\right)^{2}$. Obviously, the inequality (26) is equivalent to the condition (21). Hence, if the condition (21) is satisfied, then (26) holds and the actuator saturation constraint is achieved. Therefore, if the conditions (20)-(21) are held, then the closed-loop system (12) is asymptotically stable subject to actuator saturation (2). 
Remark 2. In Theorem 1, the sufficient conditions (20) and (21) are derived into LMI problems that can be directly calculated via optimal convex algorithm [27]. In the conditions of Theorem 1, the variables $\mathbf{P}$ and $\mathbf{K}_{i}$ are needed to be found. With increasing the number of fuzzy rules, the number of inequality conditions is increasing such that the difficulty of finding the desired variables is also increased. Thus, the computational complexity of the proposed approach will be increased when the number of fuzzy rules is arisen. For reducing the computational complexity, the nonlinear system is recommended to be modeled by T-S fuzzy model as less fuzzy rules as possible.

Remark 3. The fuzzy control problem for discrete-time nonlinear systems subject to actuator saturation has been discussed in [21]. Based on the saturation function of [21], the number of stability conditions is increased dramatically when the number of control inputs is increased. For this reason, the authors of [22] propose a novel function to formulate actuator saturation such that the energy of controller can be limited in a specific nonlinear saturation sector. Referring to [22], the number of stability conditions is not increased when the number of control inputs is increased. Based on the actuator saturation described in [22], the stability conditions of fuzzy controller design are derived in Theorem 1 for the discretetime nonlinear stochastic system with multiplicative noises.

The LMI stability conditions (20)-(21) have been derived in Theorem 1 to guarantee the stability of T-S fuzzy model with multiplicative noises subject to actuator saturation. In the following section, a truck-trailer system is proposed to demonstrate the application and usefulness of the proposed fuzzy controller design approach.

\section{Numerical Simulations for the Control of Nonlinear Truck-Trailer Systems}

Applying the proposed fuzzy controller design approach, the control problem subject to actuator saturation for a nonlinear discrete-time truck-trailer model [28] is studied in this section. The considered truck-trailer system is depicted in Figure 1. According to Figure 1, the dynamic equations of the nonlinear discrete-time truck-trailer system can be described as follows:

$$
\begin{gathered}
x_{0}(k+1)=x_{0}(k)+\frac{v \times \Delta t}{L_{1}} \tan (u(k)) \\
x_{1}(k)=x_{0}(k)-x_{2}(k) \\
x_{2}(k+1)=\frac{v \times \Delta t}{L_{2}} \sin \left(x_{1}(k)\right)+x_{2}(k) \\
x_{3}(k+1)=x_{3}(k)+v \times \Delta t \times \cos \left(x_{1}(k)\right) \\
\times \sin \left(\frac{x_{2}(k+1)+x_{2}(k)}{2}\right)
\end{gathered}
$$

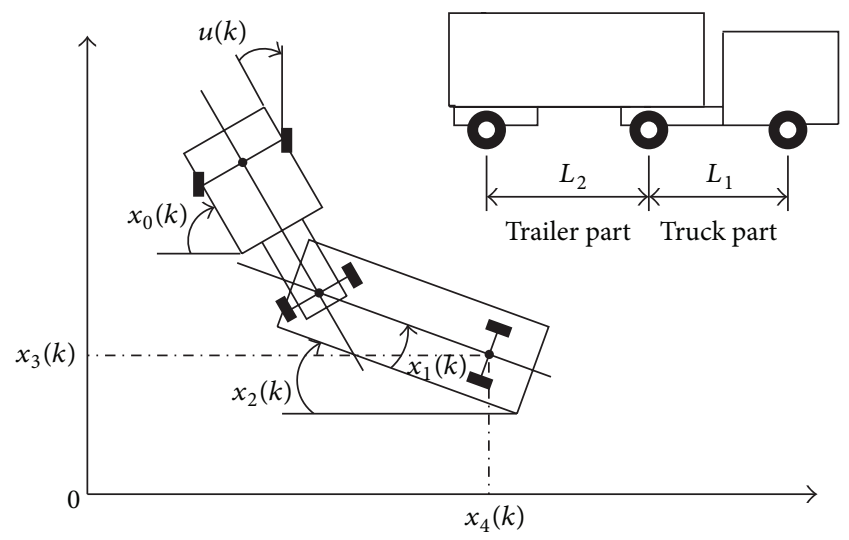

Figure 1: Nonlinear discrete-time truck-trailer system.

$$
\begin{aligned}
x_{4}(k+1)= & x_{4}(k)+v \times \Delta t \times \cos \left(x_{1}(k)\right) \\
& \times \cos \left(\frac{x_{2}(k+1)+x_{2}(k)}{2}\right),
\end{aligned}
$$

where $L_{1}$ is length of truck $(2.8 \mathrm{~m}) ; L_{2}$ is length of trailer $(5.5 \mathrm{~m}) ; \Delta t$ is sampling time $(2.0 \mathrm{sec}) ; v$ is constant speed of backing up $(-1.0 \mathrm{~m} / \mathrm{sec}) ; x_{0}(k)$ is angle of truck; $x_{1}(k)$ is angle difference between truck and trailer; $x_{2}(k)$ is angle of trailer; $x_{3}(k)$ is vertical position of rear end of trailer; $x_{4}(k)$ is horizontal position of rear end of trailer; $u(k)$ is steering angle.

For the state $x_{1}(k), 90^{\circ}$ and $-90^{\circ}$ correspond to two "jackknife" positions. The jackknife phenomenon cannot be avoided if the steering is not controlled during the backward movement. To succeed in the backing control, we need to avoid the jackknife phenomenon. The control purpose of this example is to back up a truck-trailer along straight line $\left(x_{3}=\right.$ 0 ) without forward movements as shown in Figure 1; that is, $x_{1}(k) \rightarrow 0, x_{2}(k) \rightarrow 0$, and $x_{3}(k) \rightarrow 0$.

In this example, the multiplicative noise term is added into the system for describing the stochastic behaviors. Besides, it is assumed that $x_{1}(k)$ and $u(k)$ are always small values and the horizontal position motion $x_{4}(k)$ is not considered in this example. Therefore, one can represent and simplify the original model equation ((27a), (27b), (27c), (27d), and (27e)) as follows:

$$
\begin{aligned}
x_{1}(k+1)= & \left(1-\frac{v \times \Delta t}{L_{2}}\right) x_{1}(k)+\frac{v \times \Delta t}{L_{1}} u(k)+0.01 \\
& \times\left(\left(1-\frac{v \times \Delta t}{L_{2}}\right) x_{1}(k)+\frac{v \times \Delta t}{L_{1}} u(k)\right) w(k) \\
x_{2}(k+1)= & \frac{v \times \Delta t}{L_{2}} x_{1}(k)+x_{2}(k)+0.01 \\
& \times\left(\frac{v \times \Delta t}{L_{2}} x_{1}(k)+x_{2}(k)\right) w(k)
\end{aligned}
$$




$$
\begin{aligned}
x_{3}(k+1)= & v \times \Delta t \times \sin \left(\frac{v \times \Delta t}{2 L_{2}} x_{1}(k)+x_{2}(k)\right) \\
& +x_{3}(k)+0.01 \\
& \times\left(v \times \Delta t \times \sin \left(\frac{v \times \Delta t}{2 L_{2}} x_{1}(k)+x_{2}(k)\right)\right. \\
& \left.+x_{3}(k)\right) w(k),
\end{aligned}
$$

where $w(k)$ is a scalar zero mean white noise with variance one.

Assume that $\left(v \times \Delta t / 2 L_{2}\right) x_{1}(k)+x_{2}(k)$ is operated between $(-\pi,+\pi)$. Then, the T-S fuzzy model representing the dynamics of the truck-trailer system ((28a), (28b), and (28c)) with multiplicative noise term can be described as follows. Rule 1. IF $\left(v \times \Delta t / 2 L_{2}\right) x_{1}(k)+x_{2}(k)$ is about 0 THEN

$$
x(k+1)=\mathbf{A}_{1} x(k)+\mathbf{B}_{1} \bar{u}(k)+\left(\overline{\mathbf{A}}_{1} x(k)+\overline{\mathbf{B}}_{1} \bar{u}(k)\right) w(k) .
$$

Rule 2. IF $\left(v \times \Delta t / 2 L_{2}\right) x_{1}(k)+x_{2}(k)$ is about $-\pi$ or $\pi$ THEN

$$
x(k+1)=\mathbf{A}_{2} x(k)+\mathbf{B}_{2} \bar{u}(k)+\left(\overline{\mathbf{A}}_{2} x(k)+\overline{\mathbf{B}}_{2} \bar{u}(k)\right) w(k),
$$

where

$$
\begin{aligned}
& \mathbf{A}_{1}=\left[\begin{array}{ccc}
1-\frac{v \times \Delta t}{L_{2}} & 0 & 0 \\
\frac{v \times \Delta t}{L_{2}} & 1 & 0 \\
\frac{v^{2} \times \Delta t^{2}}{2 L_{2}} & v \times \Delta t & 1
\end{array}\right] \\
& \overline{\mathbf{A}}_{1}=0.01 \times\left[\begin{array}{ccc}
1-\frac{v \times \Delta t}{L_{2}} & 0 & 0 \\
\frac{v \times \Delta t}{L_{2}} & 1 & 0 \\
\frac{v^{2} \times \Delta t^{2}}{2 L_{2}} & v \times \Delta t & 1
\end{array}\right] \text {, } \\
& \mathbf{A}_{2}=\left[\begin{array}{ccc}
1-\frac{v \times \Delta t}{L_{2}} & 0 & 0 \\
\frac{v \times \Delta t}{L_{2}} & 1 & 0 \\
\frac{\varphi \times v^{2} \times \Delta t^{2}}{2 L_{2}} & \varphi \times v \times \Delta t & 1
\end{array}\right] \\
& \overline{\mathbf{A}}_{2}=0.01 \times\left[\begin{array}{ccc}
1-\frac{v \times \Delta t}{L_{2}} & 0 & 0 \\
\frac{v \times \Delta t}{L_{2}} & 1 & 0 \\
\frac{\varphi \times v^{2} \times \Delta t^{2}}{2 L_{2}} & \varphi \times v \times \Delta t & 1
\end{array}\right] \text {, } \\
& \mathbf{B}_{1}=\mathbf{B}_{2}=\left[\begin{array}{c}
\frac{v \times \Delta t}{L_{1}} \\
0 \\
0
\end{array}\right], \quad \overline{\mathbf{B}}_{1}=\overline{\mathbf{B}}_{2}=0.01 \times\left[\begin{array}{c}
\frac{v \times \Delta t}{L_{1}} \\
0 \\
0
\end{array}\right] \text {, }
\end{aligned}
$$

and $\varphi$ is $10^{-2} / \pi$.

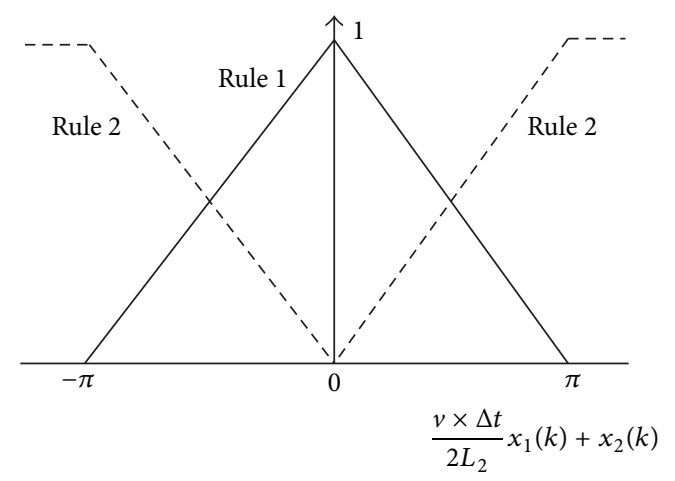

Figure 2: Membership function of $\left(v \times \Delta t / 2 L_{2}\right) x_{1}(k)+x_{2}(k)$.

The membership function is proposed in Figure 2. For applying the proposed fuzzy controller design technique, the parameters corresponding to actuator saturation are chosen as $u_{k H}=1.5$ and $\varepsilon=0.6$. Because there are two fuzzy rules in this T-S fuzzy model, three variables, that is, $\mathbf{P}, \mathbf{K}_{1}$, and $\mathbf{K}_{2}$, are needed to be found to satisfy (20) and (21). By using the LMI toolbox in MATLAB [27], the following feasible solutions can be solved:

$$
\mathbf{P}=\left[\begin{array}{ccc}
1.0467 & -1.6144 & 0.2874 \\
-1.6144 & 3.7534 & -0.6673 \\
0.2874 & -0.6673 & 0.2486
\end{array}\right]
$$

Besides, the control gains are also obtained as follows:

$$
\begin{aligned}
& \mathbf{K}_{1}=\left[\begin{array}{lll}
1.8985 & -2.0338 & 0.2439
\end{array}\right], \\
& \mathbf{K}_{2}=\left[\begin{array}{lll}
1.8589 & -1.7002 & 0.3022
\end{array}\right] .
\end{aligned}
$$

Substituting the above control gains into (10), the PDCbased fuzzy controller can be obtained. Employing the obtained fuzzy controller (10) to drive the nonlinear discretetime stochastic truck-trailer system ((28a), (28b), and (28c)), the simulation results of the state responses can be found in Figures 3, 4, and 5 with the initial conditions $x(0)=$ $\left[\begin{array}{lll}88^{\circ} & 90^{\circ} & 3\end{array}\right]^{T}$. Besides, the responses of constrained control input are shown in Figure 6. From the simulation results, one can find that the nonlinear discrete-time stochastic trucktrailer system ((28a), (28b), and (28c)) is asymptotically stable and the actuator saturation constraint is achieved.

\section{Conclusions}

In this paper, the T-S fuzzy model with multiplicative noises was employed to represent the discrete-time nonlinear stochastic systems. According to the discrete-time T-S fuzzy model with multiplicative noises, the sufficient stability conditions have been derived subject to the actuator saturation constraints. Solving these sufficient stability conditions via LMI techniques, the PDC-based fuzzy controllers can be obtained. Applying the proposed fuzzy controller design approach, some systems represented by the discrete-time T$\mathrm{S}$ fuzzy model with multiplicative noises can be protected 


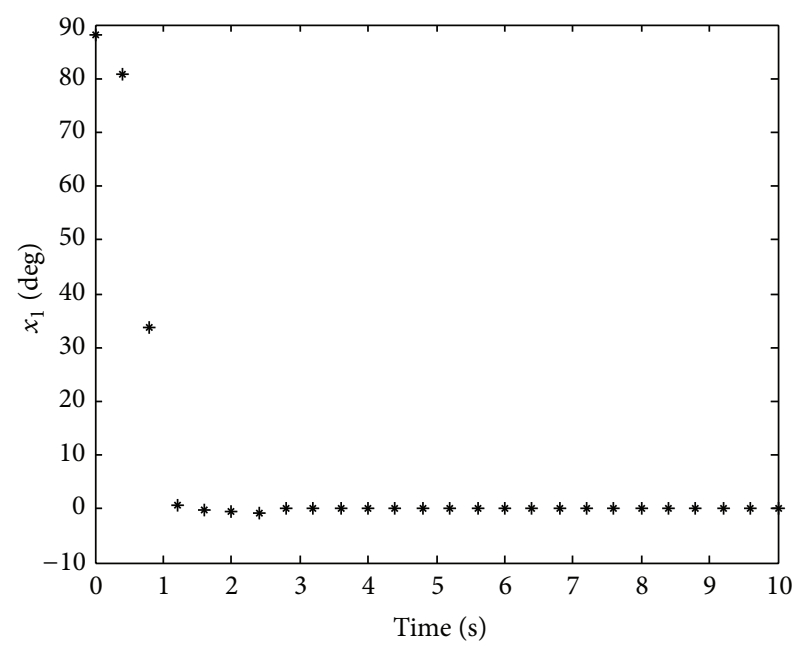

Figure 3: The angle difference $x_{1}(k)$ between truck and trailer.

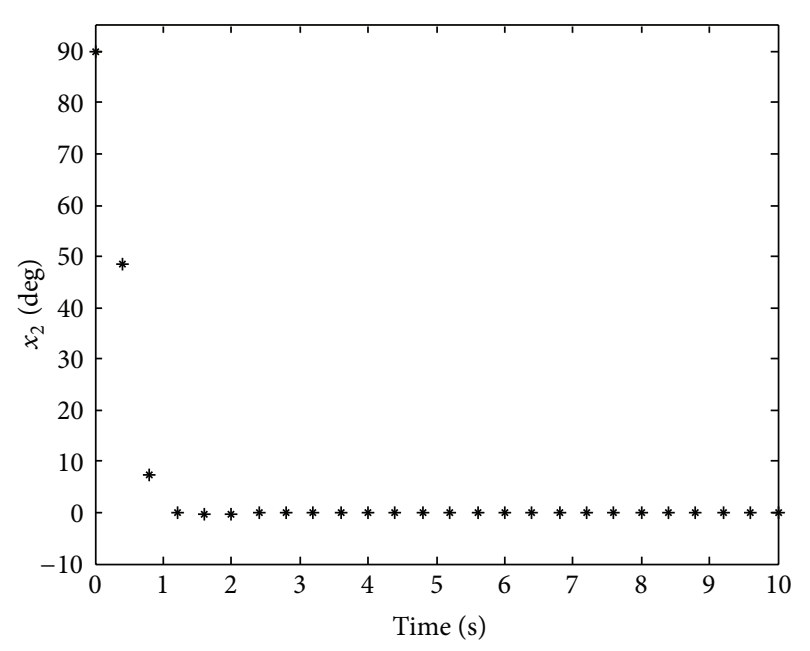

FIgURE 4: The angle $x_{2}(k)$ for trailer.

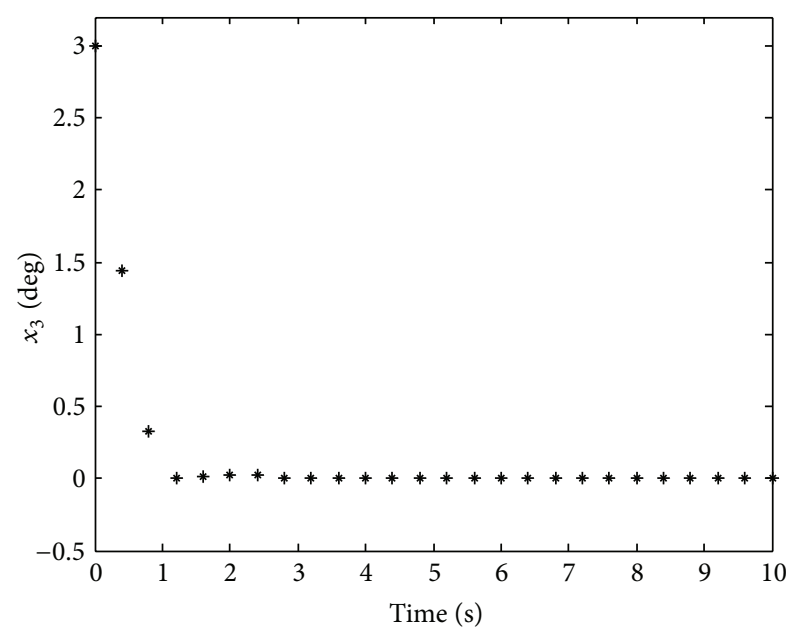

FIgURE 5: The vertical position of rear end $x_{3}(k)$ for trailer.

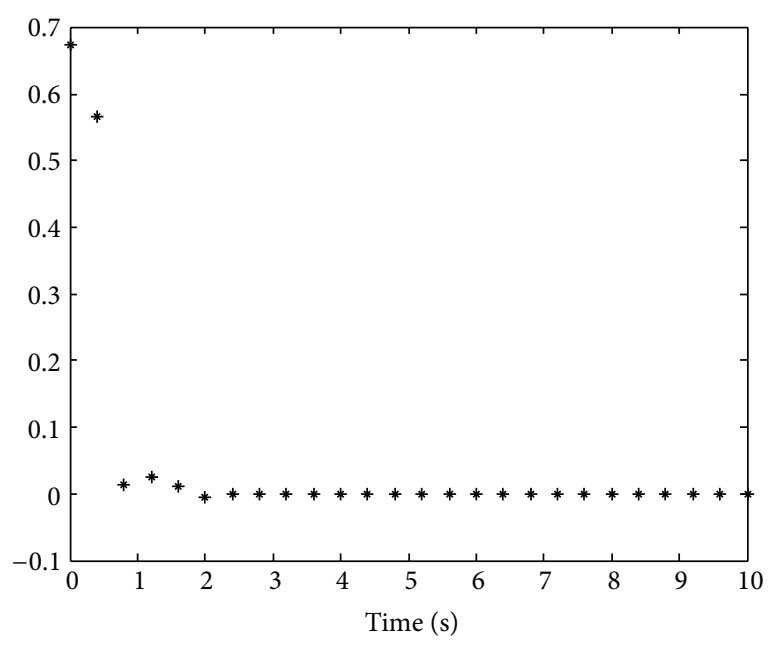

FIGURE 6: The constrained control input.

for avoiding the huge power into the precise system. Finally, a numerical simulation was provided to demonstrate the effectiveness and applicability of the proposed fuzzy control method.

\section{References}

[1] K. Tanaka and M. Sugeno, "Stability analysis and design of fuzzy control systems," Fuzzy Sets \& Systems, vol. 45, no. 2, pp. 135$156,1992$.

[2] W.-J. Chang, W.-H. Huang, W. Chang, and C.-C. Ku, "Robust fuzzy control for continuous perturbed time-delay affine Takagi-Sugeno fuzzy models," Asian Journal of Control, vol. 13, no. 6, pp. 818-830, 2011.

[3] M. R. Soltanpour, B. Zolfaghari, M. Soltani, and M. H. Khooban, "Fuzzy sliding mode control design for a class of nonlinear systems with structured and unstructured uncertainties," International Journal of Innovative Computing, Information and Control, vol. 9, no. 7, pp. 2713-2726, 2013.

[4] W. H. Ho, S. H. Chen, and J. H. Chou, "Observability robustness of uncertain fuzzy-model- based control systems," International Journal of Innovative Computing, Information and Control, vol. 9, no. 2, pp. 805-819, 2013.

[5] W.-J. Chang, C. C. Ku, and W. Chang, "Analysis and synthesis of discrete nonlinear passive systems via affine T-S fuzzy models," International Journal of Systems Science, vol. 39, no. 8, pp. 809821, 2008.

[6] W.-J. Chang, S.-S. Jheng, and C.-C. Ku, "Fuzzy control with robust and passive properties for discrete-time Takagi-Sugeno fuzzy systems with multiplicative noises," Proceedings of the Institution of Mechanical Engineers, vol. 226, no. 4, pp. 476-485, 2012.

[7] H. Zhang, Y. Shi, and M. Liu, " $H_{\infty}$ step tracking control for networked discrete-time nonlinear systems with integral and predictive actions," IEEE Transactions on Industrial Informatics, vol. 9, no. 1, pp. 337-345, 2013.

[8] H. Zhang, Y. Shi, and B. Mu, "Optimal $H_{\infty}$-based linearquadratic regulator tracking control for discrete-time takagisugeno fuzzy systems with preview actions," ASME, Journal of 
Dynamic System, Measurement, and Control, vol. 135, no. 4, pp. 044501-1-044501-5, 2013.

[9] H. Zhang, Y. Shi, and A. Saadat Mehr, "On $H_{\infty}$ filtering for discrete-time takagi-sugeno fuzzy systems," IEEE Transactions on Fuzzy Systems, vol. 20, no. 2, pp. 396-401, 2012.

[10] X. Zhang, G. Lu, and Y. Zheng, "Stabilization of networked stochastic time-delay fuzzy systems with data dropout," IEEE Transactions on Fuzzy Systems, vol. 16, no. 3, pp. 798-807, 2008.

[11] H. Zhang, Y. Wang, and D. Liu, "Delay-dependent guaranteed cost control for uncertain stochastic fuzzy systems With multiple time delays," IEEE Transactions on Systems, Man, and Cybernetics B, vol. 38, no. 1, pp. 126-140, 2008.

[12] L. Wu and D. W. C. Ho, "Fuzzy filter design for Itô stochastic systems with application to sensor fault detection," IEEE Transactions on Fuzzy Systems, vol. 17, no. 1, pp. 233-242, 2009.

[13] L. Wu and W. X. Zheng, " $L_{2}-L_{\infty}$ control of nonlinear fuzzy itô stochastic delay systems via dynamic output feedback," IEEE Transactions on Systems, Man, and Cybernetics B, vol. 39, no. 5, pp. 1308-1315, 2009.

[14] C.-C. Ku, P.-H. Huang, and W.-J. Chang, "Passive fuzzy controller design for nonlinear systems with multiplicative noises," Journal of the Franklin Institute, vol. 347, no. 5, pp. 732-750, 2010.

[15] W.-J. Chang, C.-C. Ku, and P.-H. Huang, "Robust fuzzy control for uncertain stochastic time-delay Takagi-Sugeno fuzzy models for achieving passivity," Fuzzy Sets \& Systems, vol. 161, no. 15, pp. 2012-2032, 2010.

[16] W.-J. Chang, C.-C. Ku, and C.-H. Chang, "PDC and non-PDC fuzzy control with relaxed stability conditions for contintuoustime multiplicative noised fuzzy systems," Journal of the Franklin Institute, vol. 349, no. 8, pp. 2664-2686, 2012.

[17] B.-S. Chen and S. S. Wang, "The stability of feedback control with nonlinear saturating actuator: time domain approach," IEEE Transactions on Automatic Control, vol. 33, no. 5, pp. 483487, 1988.

[18] W. J. Wang and B.-S. Chen, "Stability of large-scale systems with saturating actuators," International Journal of Control, vol. 47, no. 3, pp. 827-850, 1988.

[19] Y.-Y. Cao and Z. Lin, "Robust stability analysis and fuzzyscheduling control for nonlinear systems subject to actuator saturation," IEEE Transactions on Fuzzy Systems, vol. 11, no. 1, pp. 57-67, 2003.

[20] W.-J. Chang and S.-M. Wu, "Continuous fuzzy controller design subject to minimizing control input energy with output variance constraints," European Journal of Control, vol. 11, no. 3 , pp. 269-279, 2005.

[21] S. Lee, E. Kim, H. Kim, and M. Park, "Robust analysis and design for discrete-time nonlinear systems subject to actuator saturation via fuzzy control," IEICE Transactions on Fundamentals of Electronics, Communications and Computer Sciences, vol. E88-A, no. 8, pp. 2181-2191, 2005.

[22] C.-S. Tseng and B.-S. Chen, " $H_{\infty}$ fuzzy control design for nonlinear systems subject to actuator saturation," in Proceedings of the IEEE International Conference on Fuzzy Systems, pp. 783788, Canada, July 2006.

[23] X. Gao, M. Ma, and H. Chen, "Guaranteed cost tracking scheme for wheeled mobile robot with actuator saturations via T-S fuzzy model," in Proceedings of the 5th International Conference on Fuzzy Systems and Knowledge Discovery (FSKD '08), pp. 85-89, Jinan, China, October 2008.
[24] T. Zhang, G. Feng, H. Liu, and J. Lu, "Piecewise fuzzy antiwindup dynamic output feedback control of nonlinear processes with amplitude and rate actuator saturations," IEEE Transactions on Fuzzy Systems, vol. 17, no. 2, pp. 253-264, 2009.

[25] K. Tanaka and H. O. Wang, Fuzzy Control Systems Design and Analysis: A Linear Matrix Inequality Approach, John Wiley \& Sons, 2001.

[26] S. Boyd, L. El Ghaoui, E. Feron, and V. Balakrishnan, Linear Matrix Inequalities in System and Control Theory, SIAM, Philadelphia, Pa, USA, 1994.

[27] P. Gahinet, A. Nemirovski, A. J. Laub, and M. Chilali, LMI Control Toolbox, The MathWorks, Natick, Mass, USA, 1995.

[28] K. Tanaka and M. Sano, "Robust stabilization problem of fuzzy control systems and its application to backing up control of a truck-trailer," IEEE Transactions on Fuzzy Systems, vol. 2, no. 2, pp. 119-134, 1994. 


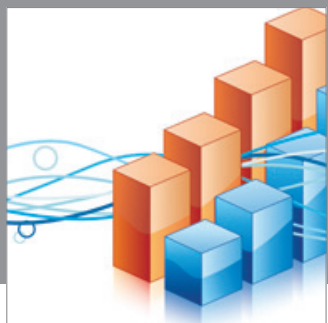

Advances in

Operations Research

mansans

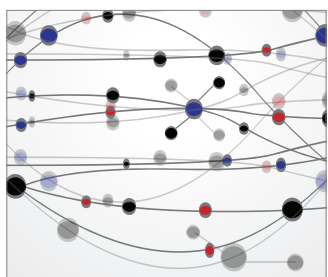

The Scientific World Journal
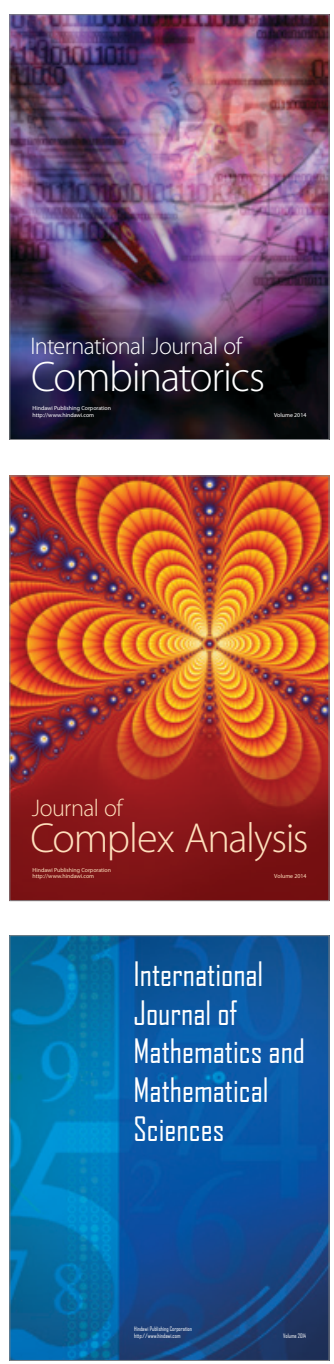
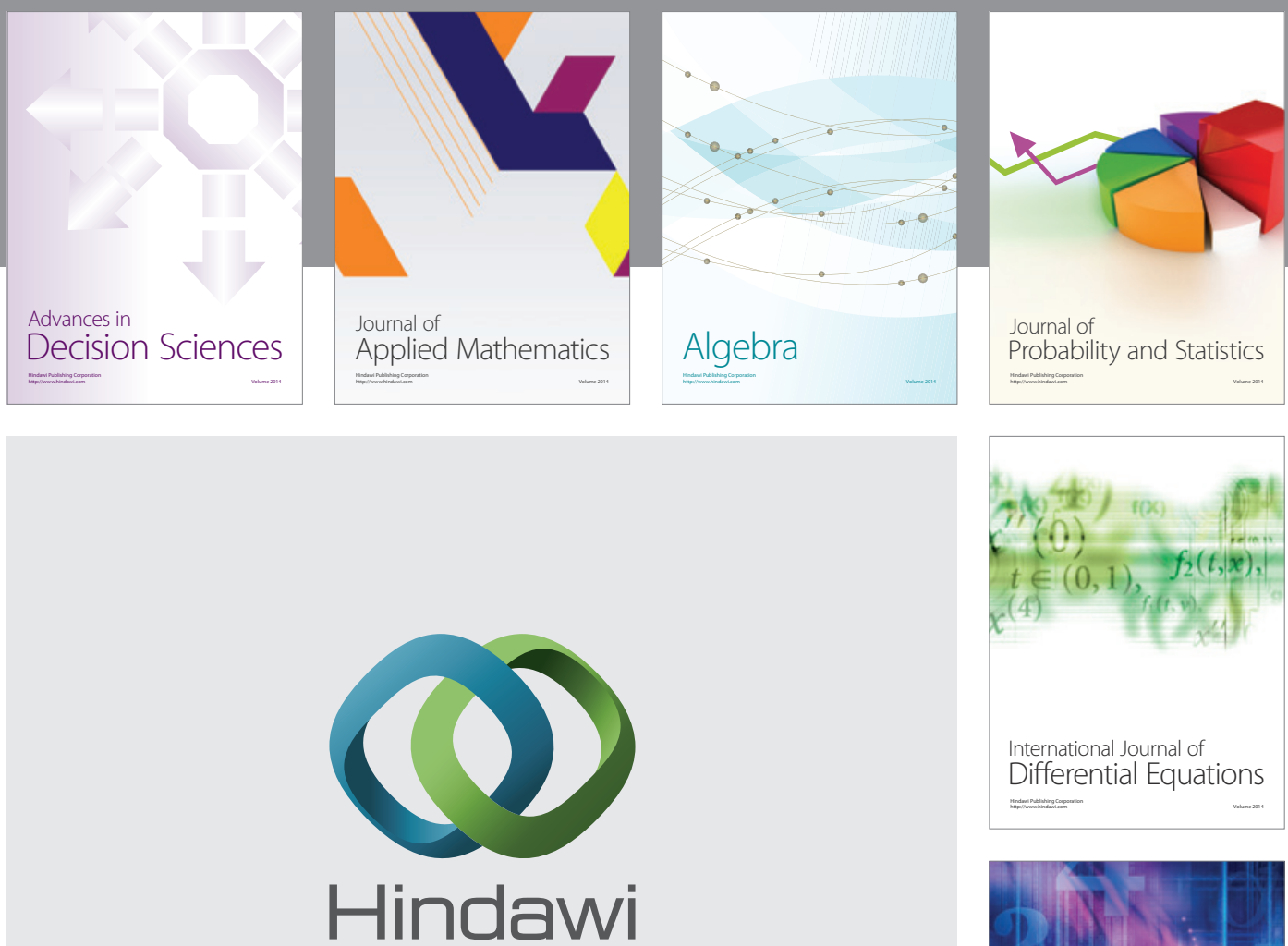

Submit your manuscripts at http://www.hindawi.com
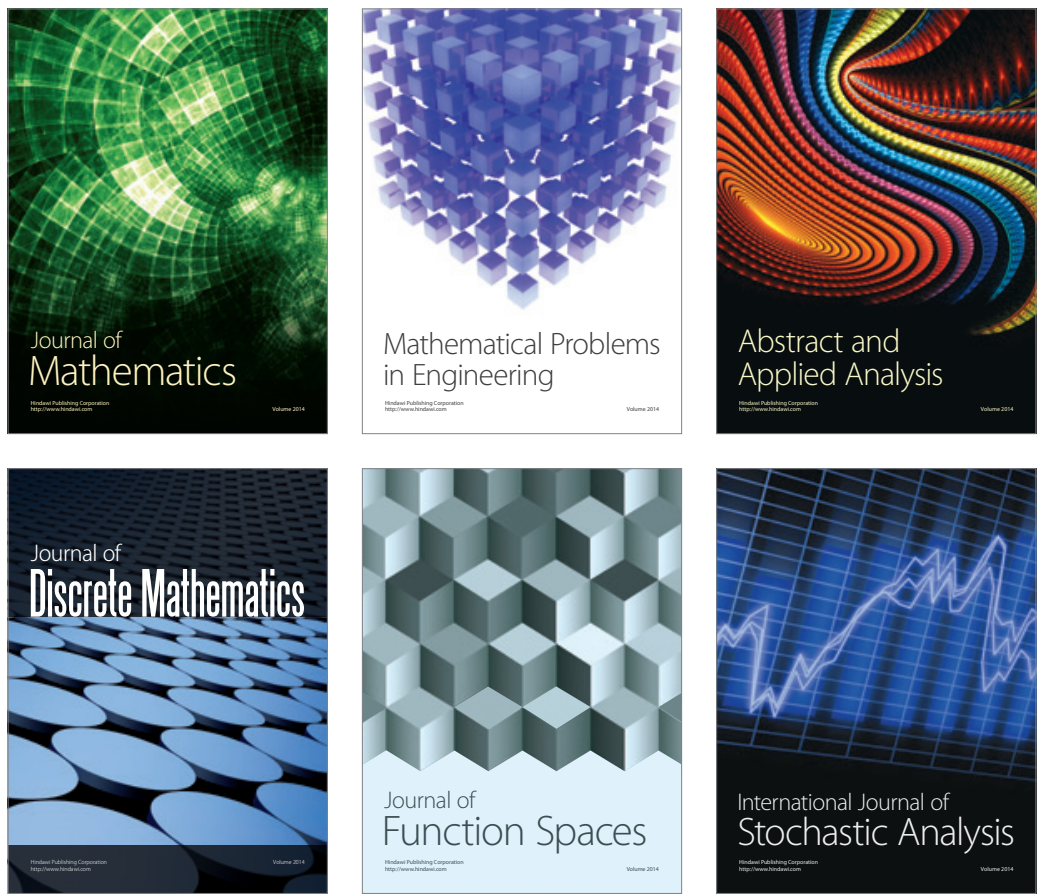

Journal of

Function Spaces

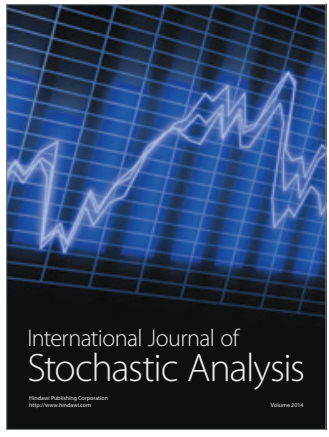

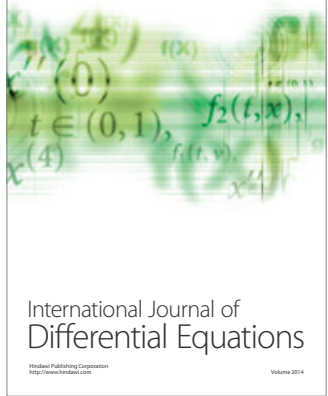
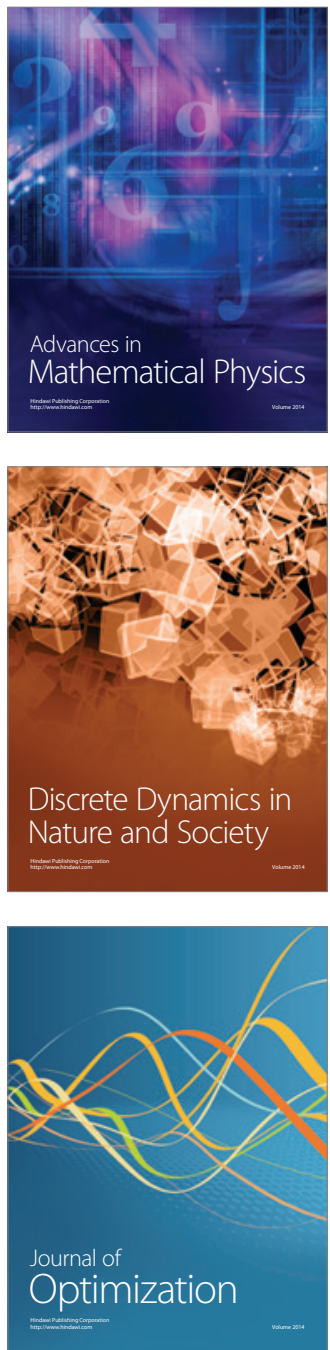